\title{
Biliverdin and bilirubin sulfonate inhibit monosodium urate induced sterile inflammation
}

in the rat

Ryan G. Shiels ${ }^{1,2}$, Wenu Hewage ${ }^{1,2}$, Evan N. Pennell ${ }^{1,2}$, Josif Vidimce ${ }^{1,2}$, Gary Grant ${ }^{1}$, Andrew G. Pearson ${ }^{1,2}$, Karl-Heinz Wagner ${ }^{3}$, Michael Morgan ${ }^{4}$ and Andrew C. Bulmer ${ }^{1,2^{*}}$

${ }^{1}$ School of Medical Science, Griffith University, Gold Coast, Queensland, Australia

${ }^{2}$ Menzies Health Institute Queensland, Griffith University, Gold Coast, Queensland, Australia

${ }^{3}$ Department of Nutritional Sciences, University of Vienna, Vienna, Austria

${ }^{4}$ Department of Anatomy and Neuroscience, University of Melbourne, Melbourne, Australia

\begin{abstract}
Background: Biliverdin, a by-product of haem catabolism, possesses potent endogenous antioxidant and anti-inflammatory properties. Bilirubin-C10-sulfonate (BRS), an active metabolite formed after enteral administration of BV in the rat, also possess antioxidant properties. Therefore, we investigated the anti-inflammatory and antioxidant activity of BV and BRS in an in vivo model of monosodium urate induced sterile inflammation.
\end{abstract}

Methods: Subcutaneous air pouches were created on the dorsal flanks of Wistar rats (10-12 weeks of age). Prior to stimulation of the 6-day old pouch with monosodium urate $(25 \mathrm{mg})$, groups were pre-treated with intraperitoneal BRS $(27 \mathrm{mg} / \mathrm{kg})$ and BV $(27 \mathrm{mg} / \mathrm{kg})$. Total and differential leukocyte counts were determined in pouch fluid aspirate at 1, 6, 12, 24 and 48 $\mathrm{h}$ after monosodium urate stimulation. Biliverdin (BV), BRS and unconjugated bilirubin (UCB) concentrations in the serum and pouch fluid were quantified using liquid chromatographymass spectrometry. Pouch fluid cytokine concentrations (IL-1 $\beta$, IL-1 $\alpha$, TNF- $\alpha$, IL-17A, IL-12, GM-CSF, IL-33, IFN- $\gamma$, IL-18, IL-10, MCP-1, CXCL-1 and IL-6) were assessed after $6 \mathrm{~h}$. In addition, $24 \mathrm{~h}$ protein carbonyl and chloramine concentrations were assessed in pouch fluid using ELISA and spectrophotometry, respectively.

Results: BRS and BV significantly $(p<0.05)$ inhibited leukocyte (total, neutrophil and macrophage) infiltration into the pouch fluid from 6 to $48 \mathrm{~h}$. For example, after $6 \mathrm{~h}$ neutrophil counts decreased following BRS $\left(0.32 \pm 0.11 \times 10^{6}\right.$ cells $\left.\mathrm{mL}^{-1}\right)$ and BV $(0.17 \pm 0.03$ $\times 10^{6}$ cells $\mathrm{mL}^{-1}$ ) compared to MSU only $\left(3.51 \pm 1.07 \times 10^{6}\right.$ cells $\left.\mathrm{mL}^{-1}\right)$. Both BV and BRS significantly $(\mathrm{p}<0.05)$ reduced pouch GM-CSF (BV: $5.8 \pm 1.2 \mathrm{pg} \mathrm{mL}^{-1}$, BRS: $6.9 \pm 1.5 \mathrm{pg} \mathrm{mL}^{-1}$ vs MSU only: $13.0 \pm 1.9 \mathrm{pg} \mathrm{mL}^{-1}$ ) and MCP-1 concentrations at $6 \mathrm{~h}$ (BV: $1804 \pm 269 \mathrm{pg} \mathrm{mL}^{-1}$, BRS: $7927 \pm 2668 \mathrm{pg} \mathrm{mL}^{-1} \mathrm{vs} \mathrm{MSU}$ only: $17290 \pm 4503 \mathrm{pg} \mathrm{ml}^{-1}$ ), whilst BV additionally inhibited IL-6 (4354 $\pm 977 \mathrm{pg} \mathrm{mL}^{-1}$ vs MSU only: $\left.25070 \pm 5178 \mathrm{pg} \mathrm{mL}^{-1}\right)$ and IL-18 (17.6 \pm 2.0 pg mL $\mathrm{mL}^{-1}$ vs MSU only: $\left.81.5 \pm 19.9 \mathrm{pg} \mathrm{mL}^{-1}\right)$ concentrations at $6 \mathrm{~h}(\mathrm{p}<0.05)$. Despite these differences, no change in pouch chloramine or protein carbonyl concentrations occurred at $24 \mathrm{~h}(\mathrm{p}>0.05)$. Serum BV concentrations rapidly diminished over $6 h$, however, BRS was readily detected in the serum over $48 \mathrm{~h}$, and in pouch fluid over $12 \mathrm{~h}$.

Conclusions: This study is the first to elucidate anti-inflammatory activity of BRS and the efficacy of BV administration in a model of gouty inflammation. Reduced leukocyte infiltration and cytokine production in response to sterile inflammation further support the 
importance of these molecules in physiology and their therapeutic potential in sterile inflammation. 


\section{Introduction}

Tetrapyrroles are involved in cellular metabolic processes including respiration and photosynthesis, and are evolutionarily conserved across all kingdoms of life. ${ }^{(1)}$ From a precursor for phycobilin synthesis in cyanobacteria and algae ${ }^{(2)}$, to a part of the haem catabolic pathway/anti-inflammatory mechanism in mammals ${ }^{(3,4)}$, these tetrapyrroles appear to be vital for the survival of all organisms that synthesise them.

Tetrapyrrolic bile pigments such as biliverdin (BV) and unconjugated bilirubin (UCB) are formed during haem catabolism following red blood cell breakdown, an event associated with acute tissue injury. ${ }^{(5)}$ The innate inflammatory responses to injury occurs in the early phases of tissue injury, however tissue injury is accompanied by the production of BV and $U C B$, which attenuates the subsequent inflammatory response and improves wound healing. ${ }^{(6)}$ Additionally, Gilbert's Syndrome (GS) is a condition presenting with a mildly elevated circulating UCB concentration caused by a reduced uridine glucuronosyltransferase $1 \mathrm{~A} 1$ (UGT1A1) deficiency due to a polymorphism ( \%60 reduction in function), and is associated with protection against oxidative processes underpinned by a greater antioxidant capacity in plasma which contributes to the $10.1 \%$ lower incidence of cardiovascular disease within the GS population. ${ }^{(7-11)}$

Administration of BV protects from inflammatory and oxidant-mediated damage in vivo. ${ }^{(12-}$ 15) Specifically, BV administration protects via reducing the expression or release of inflammatory cytokines IL-6, MCP-1 and TNF- $\alpha$ in models of ischemia-reperfusion injury. ${ }^{13 \text {, }}$ 16, 17) Additionally, BV protects from Forssmann reagent induced anaphylaxis ${ }^{(18)}$, tissue transplantation ${ }^{(15)}$, and endotoxin-induced acute lung injury ${ }^{(14)}$ with a recent study demonstrating that $\mathrm{BV}$ and related bile pigments neutralise the harmful effects of superoxide. ${ }^{(19)}$ Interestingly, intraduodenal (i.d.) and intraperitoneal (i.p.) administration of $\mathrm{BV}$ in rodents also leads to the formation of bilirubin-10-sulfonate $(B R S)^{(20,21)}$, a novel antioxidant with the potential to confer protection against oxidative and, potentially, inflammatory processes. ${ }^{(20)}$ Testing the anti- inflammatory therapeutic potential of these bile pigments clearly requires further in vivo investigation in models of sterile inflammation with clinical relevance to human inflammatory conditions in order to determine their potential physiological importance and their suitability as novel therapeutics. Gouty arthritis 
is a common clinically relevant sterile inflammatory condition, with many patient's disease being refractory to treatment. In the United States alone, the financial burden of gouty arthritis in 2013 was $\$ 1$ billion, with acute gouty arthritis attacks accounting for $32 \%$ of this cost. ${ }^{(22)}$ New treatments could potentially aid in the treatment of this condition, however, it remains unknown whether bile pigments can influence the course of this condition or its pathophysiology.

MSU crystals can activate the NLRP3 inflammasome via lysosomal leakage of cathepsins into the cytosol. ${ }^{(23)}$ Furthermore, it is well documented that MSU crystals induce inflammatory cytokine and chemokine production ${ }^{(24,25)}$ and initiate an acute inflammatory response mimicking the condition of gout in humans. ${ }^{(25,26)}$ This response is likely induced by multiple mechanisms, including direct activation of leukocytes, membrane lysis and complement activation. ${ }^{(27)}$ During the acute phase of the inflammatory response, inflammatory cytokines monocyte chemotactic protein-1 (MCP-1), interferon-gamma (IFN- $\gamma$ ), interleukin-6 (IL-6), interleukin-1 $\beta$ (IL-1 $\beta$ ), myeloperoxidase (MPO), macrophage inflammatory protein-1 $\alpha$ (MIP$1 \alpha)$, tumour necrosis factor- $\alpha(\mathrm{TNF}-\alpha)$, chemokine ligand 1 and 2 (CXCL-1 and CCL-2 respectively), interleukin-8 (IL-8), and macrophage inflammatory protein-2 (MIP-2) have been reported to increase in MSU crystal air pouch inflammation models. ${ }^{(24)}$ Bile pigments attenuate MCP-1, IL-6 and MPO responses, therefore, it was hypothesised that BRS and BV administration to the MSU subcutaneous air pouch model of inflammation would significantly reduce both leukocyte infiltration, inflammatory cytokines and biomarkers of oxidative stress/modifications within the inflammatory milieu of the pouch. 


\section{Materials and Methods}

\section{Materials}

Biliverdin hydrochloride and bilirubin sulfonate were obtained from Frontier Scientific (Utah, USA). All other reagents were obtained from Sigma-Aldrich (Australia), unless otherwise stated.

\section{Sodium biliverdinate synthesis}

$50.07 \mathrm{mg}$ of $\mathrm{NaOH}$ (1.252 mmol) was added to $400 \mathrm{~mL}$ of ethanol and sonicated for $10 \mathrm{~min}$ at $32^{\circ} \mathrm{C}$. This solution was then added to a flask containing $250 \mathrm{mg}$ of $\mathrm{BV}-\mathrm{HCl}(0.404 \mathrm{mmol})$. This solution was again sonicated for $10 \mathrm{~min}$ at $32^{\circ} \mathrm{C}$, then $20 \mathrm{~mL}$ aliquots of the $0.625 \mathrm{mg}$ $\mathrm{mL}^{-1}$ BV solution were transferred to $50 \mathrm{~mL}$ Falcon tubes, with each containing $12.5 \mathrm{mg}$ of BV. These solutions were then evaporated to dryness (Speedyvac centrifuge vacuum concentrator; LabGear, Australia) at $400 \times \mathrm{g}, 32^{\circ} \mathrm{C}$ for $12 \mathrm{~h}$ at a pressure of 2 millibar. The dry aliquots were sealed, transferred to a $-80^{\circ} \mathrm{C}$ freezer and reconstituted in PBS immediately prior to use. All solutions were protected from light during preparation using aluminium foil and a darkened laboratory.

\section{Monosodium Urate Crystal synthesis}

Monosodium urate crystals were synthesised using previously published methods. ${ }^{(28)}$ Uric acid (1.680 g; Abcam, United Kingdom) was added to $400 \mathrm{~mL}$ of double distilled $\mathrm{H}_{2} \mathrm{O}$ and stirred at 15 RPM for $5 \mathrm{~s}$. Then, $2.25 \mathrm{~mL}$ of $5 \mathrm{M} \mathrm{NaOH}$ was added and the solution was stirred at 15 RPM for $5 \mathrm{~s}$. The solution was then heated to $60^{\circ} \mathrm{C}$, and then left at room temperature for $24 \mathrm{~h}$. Following this, the solution was filtered under light vacuum (grade 5 Whatman paper, Thomas Scientific, USA) and the resulting crystalline solids were washed with $200 \mathrm{~mL}$ of $100 \%$ ethanol before being dried under vacuum. The crystals were observed via Scanning Electron Microscopy (SEM) at $15 \mathrm{kV}$ under high vacuum with images taken at 10 random fields of view $(15 \mathrm{kV} \times 1000)$. In each field, 10 crystals were counted, and their respective length and width quantified (Neoscope JCM-5000, Jeol, Japan) and were observed to have the characteristic needle-like appearance and dimensions observed in clinical gout (length: $11.4 \pm 5.52 \mu \mathrm{m}, \mathrm{n}=100$, width: $2.09 \pm 0.73 \mu \mathrm{m}, \mathrm{n}=100)^{(28,29)}$, (Figure 1). MSU crystals were gently pressed and distributed over electrical tape and loaded onto a 
sample stub, which was then coated with gold for 2 min using a NeoCoater (MP-19020NCTR, Jeol, Japan). Administered MSU crystals were tested via limulus amebocyte lysate kit and were endotoxin free (<0.03 $\mathrm{EU} \mathrm{mg}^{-1}$; Genscript, USA).

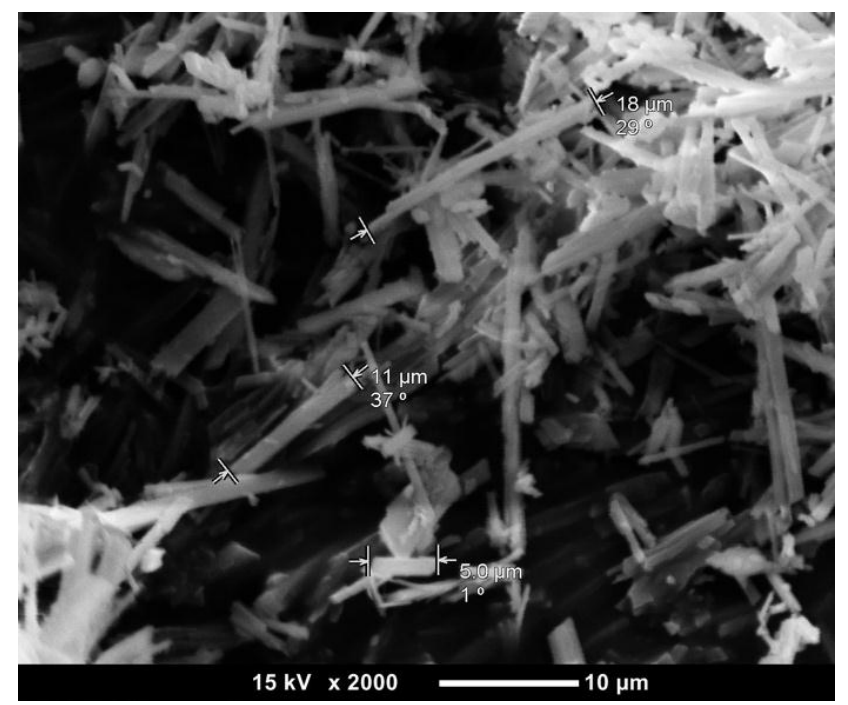

Figure 1: A representative scanning electron microscope image of monosodium urate crystals used to induce sterile inflammation within the air pouch model.

\section{Sterile Inflammation Air Pouch Model}

Adult male Wistar rats (10-12 weeks of age; 344-440 g) were housed at constant temperature, under a $12 \mathrm{~h}$ light-dark cycle and with free access to food and water. Prior to each procedure, animals were anaesthetised using 3\% isoflurane (Pharmachem, Australia), at $1 \mathrm{~L} \mathrm{~min}{ }^{-1}$ in $100 \%$ oxygen and maintained under anaesthesia via nose cone. Initially, the animals' dorsal flanks were shaved, and subcutaneous air pouches were formed immediately below the scapulae via subcutaneous delivery of $20 \mathrm{~mL}$ of sterile filtered air ( $0.22 \mu \mathrm{m}$ syringe filter and a $27 \mathrm{G}$ needle). On the fourth day, a further $10 \mathrm{~mL}$ of filtered air was delivered to the pouch to maintain patency and assist the formation of a pseudosynovial joint capsule. $^{(30)}$

On the sixth day, under light anaesthesia, $5 \mathrm{~mL}$ of a $5 \mathrm{mg} \mathrm{mL}^{-1}$ sterile suspension of MSU crystals in $0.9 \% \mathrm{NaCl}$ (Baxtor, Australia) was injected into the air pouch of each rat via a $21 \mathrm{G}$ needle, except for the sham group $(n=3)$, which received only $5 \mathrm{~mL}$ of saline. Thirty min prior to MSU delivery, rats received a $3.5 \mathrm{~mL}$ i.p. injection of $B R S\left(27 \mathrm{mg} \mathrm{kg}^{-1}, \mathrm{n}=6\right), \mathrm{BV}\left(27 \mathrm{mg} \mathrm{kg}^{-1}\right.$, $\mathrm{n}=5$, dissolved 1:1 in PBS: $\mathrm{NaCl}$ ), ibuprofen (positive control; $8.1 \mathrm{mg} \mathrm{kg}^{-1}, \mathrm{n}=5$; Glentham Life Sciences, United Kingdom) or PBS (MSU only and sham groups) through a $5 \mathrm{~mL}$ syringe and 
$0.45 \mu \mathrm{M}$ syringe filter. The dose of BV and BRS were chosen following a recent pharmacokinetic study ${ }^{(21)}$ and the dose of ibuprofen was chosen as it represents molar equivalence with BV. All compounds were dissolved in PBS and injected with a $25 \mathrm{G}$ needle unless otherwise stated.

Samples of pouch exudate were taken under light anaesthesia periodically at $1,6,12,24$, and $48 \mathrm{~h}$ after MSU administration by injecting $5 \mathrm{~mL}$ of sterile saline into the pouch, gently massaging the pouch and withdrawing $4 \mathrm{~mL}$ of exudate which was collected into a $4 \mathrm{~mL}$ EDTA tube (5.3 mM EDTA; BD, USA) and stored on ice. Additionally, $200 \mu \mathrm{L}$ of blood was collected via a tail vein bleed at each timepoint. Leukocyte counts were estimated via hemocytometer, and the remaining fluid along with the blood sample were centrifuged for $8 \mathrm{~min}$ at $2000 \times g$ at room temperature (RT). The supernatant and serum were aliquoted, placed on dry ice and stored at $-80^{\circ} \mathrm{C}$, and cell pellets were used for differential staining using a Rapid Diff stain (Australian Biostain, Australia) according to the manufacturer's instructions.

\section{Inflammatory markers}

Cytokines (IL-1 $\beta$, IL-1 $\alpha$, TNF- $\alpha$, IL-17A, IL-12, GM-CSF, IL-33, IFN- $\gamma$, IL-18, IL-10, MCP-1, CXCL1 and IL-6) were quantified in the $6 \mathrm{~h}$ pouch exudate using a bead-based immunoassay sandwich ELISA method (LEGENDplex, Biolegend, USA). Samples were processed quantification of soluble analytes performed utilising a BD SORP LSR II Fortessa flow cytometer, as per the manufacturer's instructions for use (IFU). A minimum of 500 events per analyte was acquired in the classification channel fluorophore APC (Ex 640nm Em: 670/30) with Median Fluorescence Intensity (MFI) of the reported fluorophore (PE; Ex 561 Em 588/12) interpolated against relevant standard curves using the proprietary Biolegend Software (LEGENDplex v8.0).

\section{Biomarkers of oxidative stress/modification}

Protein carbonyl concentration in pouch fluid was assessed at $24 \mathrm{~h}$ via ELISA (Biocell, New Zealand) and chloramine concentration was assessed using previously published methods. ${ }^{(31)}$ Protein concentration was assessed by Pierce BCA kit (Thermofisher, Australia). Myleoperoxidase (MPO) was assessed at 6, 12 and $24 \mathrm{~h}$ via ELISA (resolvingIMAGES, 
Australia), however, the values were below the sensitivity of the assay and are not presented.

\section{Bile pigment quantification}

Serum samples were analysed for bile pigments via quantitative UHPLC-MS (LCMS-2020, Shimadzu, Japan). Separation of the targeted tetrapyrroles was achieved using a reverse phase C18 column (Vision HT C18HL, $2 \mathrm{~mm} \times 100 \mathrm{~mm}, 1.5 \mu \mathrm{m}$, PhaseSep, Australia). The UHPLC column was preceded by a VisionHT C18HL guard column $(2 \mathrm{~mm} \times 5 \mathrm{~mm}, 1.5 \mu \mathrm{m}$, PhaseSep, Australia) and an UltraShield UHPLC precolumn filter (Restek, $0.2 \mu \mathrm{m}$, Shimadzu, Australia), respectively. The column oven and autosampler were set to $45^{\circ} \mathrm{C}$ and $4{ }^{\circ} \mathrm{C}$, respectively, and the flow rate was $0.35 \mathrm{~mL} \mathrm{~min}^{-1}$.

\section{Analysis of bile pigments in serum and pouch exudate}

The initial mobile phase consisted of $34 \%$ mobile phase B (HPLC grade methanol, Scharlau, Spain) and $66 \%$ mobile phase A (10 mM ammonium acetate in $25 \%$ HPLC grade methanol and $75 \%$ Milli-Q $\mathrm{H}_{2} \mathrm{O}$ ), representing a $50.5 \%$ initial methanol concentration. The gradient program applied for the analytical portion of the analysis was as follows: $0.3 \mathrm{~min}-50 \% \mathrm{~B}$; $1.5 \mathrm{~min}-74 \% \mathrm{~B} ; 1.8 \mathrm{~min}-92 \% \mathrm{~B} ; 3.1 \mathrm{~min}-92 \% \mathrm{~B}$. The re-equilibration portion of the analysis ran from $3.1 \mathrm{~min}$ to $6.5 \mathrm{~min}$ at $34 \% \mathrm{~B}$, with the flow rate increased to $0.42 \mathrm{~mL} \mathrm{~min}^{-1}$ at $3.5 \mathrm{~min}$, held until $4.0 \mathrm{~min}$, reducing linearly to $0.35 \mathrm{~mL} \mathrm{~min}^{-1}$ at $4.4 \mathrm{~min}$ and remaining so until $6.5 \mathrm{~min}$. The total run time including re-equilibration was $6 \mathrm{~min}$, with retention times of $2.00,2.25$ and 2.76 min for BRS, BV and $U C B$, respectively. A $2 \mu \mathrm{L}$ injection volume was used for all analyses. ESI operating conditions were: desolvation line temperature $250^{\circ} \mathrm{C}$, heat block temperature $200^{\circ} \mathrm{C}$, nebulizing gas flow $1.5 \mathrm{~L} \mathrm{~min}^{-1}\left(\mathrm{~N}_{2}\right)$, drying gas flow $15 \mathrm{~L} \mathrm{~min}{ }^{-1}$ with all detection voltages set via autotune using the manufacturer's tuning solution (Shimadzu, Japan). Data were collected in negative ion mode via single ion monitoring (SIM) of the $\mathrm{m} / \mathrm{z}$ corresponding with the deprotonated cation $[\mathrm{M}-\mathrm{H}]^{-}$of each compound.

\section{External and Internal Quantification Standards}

Stock solutions of BRS, BV and UCB were dissolved individually at $8 \mathrm{mM}$ in DMSO, then combined with additional DMSO to form a mixed stock solution containing $2 \mathrm{mM}$ of each 
compound. This mixed stock solution was further diluted with DMSO in order to form 'working solutions' with concentrations from $390 \mathrm{nM}$ to $200 \mu \mathrm{M}$ and were made fresh prior to each standard curve.

Internal standards (IS) included mesobilirubin-10-sulfonate, mesobiliverdin and mesobilirubin (mBRS, mBV and mUCB, respectively; Frontier Scientific, USA). A mixed IS solution was created by initially dissolving each IS at $8 \mathrm{mM}$ in DMSO, which were then combined and further diluted with DMSO to form the final IS solution with $\mathrm{mBRS} / \mathrm{mBV} / \mathrm{mUCB}$ concentrations of $80 / 80 / 40 \mu \mathrm{M}$, respectively. Aliquots of $500 \mu \mathrm{L}$ of the IS solutions were made, and frozen in liquid nitrogen prior to storage at $-80^{\circ} \mathrm{C}$. Prior to use, IS aliquots were defrosted in darkness at room temperature.

\section{Calibration}

Matrix matched calibration standards were prepared by adding $10 \mu \mathrm{L}$ of 'working solution' to $390 \mu \mathrm{L}$ of fresh rat serum to form spiked serum containing BRS/BV/UCB at concentrations from $\sim 391 \mathrm{nM}$ to $200 \mu \mathrm{M}$ plus one DMSO only blank. After $10 \mathrm{~s}$ of mixing via vortex and 10 min of incubation in the dark at room temperature, $40 \mu \mathrm{L}$ of spiked serum was added to 10 $\mu \mathrm{L}$ of IS solution followed by mixing for $5 \mathrm{~s}$ via vortex. To this solution, $150 \mu \mathrm{L}$ of 1:4 DMSO:methanol was added and mixed via vortex again for $10 \mathrm{~s}$ before being centrifuged $(21500 \times \mathrm{g} ; 10 \mathrm{~min})$. Prior to injection, the supernatant was passed through a $0.22 \mu \mathrm{m}$ syringe filter (PhaseSep, Australia) and $2 \mu \mathrm{L}$ of the filtrate was injected for analysis.

\section{Quality control}

Quality control (QC) standards were prepared as per the calibration standards above at BRS/BV/UCB concentrations of 1.56/12.5/50 $\mu \mathrm{M}$ representing a low/medium/high concentration, and $40 \mu \mathrm{L}$ of each were aliquoted into $1.5 \mathrm{~mL}$ microtubes. Prior to flash freezing in liquid $\mathrm{N}_{2}$ and storage at $-80^{\circ} \mathrm{C}, 10 \mu \mathrm{L}$ of IS was spiked into each aliquot of rat serum spiked with mixed standard and vortexed for $10 \mathrm{~s}$. Prior to each batch of sample analysis, one aliquot of each concentration level was defrosted while shielded from light, prepared as above and injected for analysis to confirm accuracy within acceptance criteria range of $85-115 \%$. Following every $10^{\text {th }}$ sample injection, one QC standard was reanalysed to ensure assay validity over time. 


\section{Sample analysis}

Prior to serum analysis, $10 \mu \mathrm{L}$ of IS solution was added to $40 \mu \mathrm{L}$ of sample serum or pouch exudate, mixed via vortex for $10 \mathrm{~s}$ before adding $150 \mu \mathrm{L}$ of 1:4 DMSO:methanol and mixing via vortex again for $10 \mathrm{~s}$ before being centrifuged $(21500 \times g ; 10 \mathrm{~min})$. The supernatant was then passed through a $0.22 \mu \mathrm{m}$ syringe filter (PhaseSep, Australia) and $2 \mu \mathrm{L}$ of the resulting filtrate was injected for analysis.

Concentrations of tetrapyrroles in serum were calculated via the internal standard method using AUC integration of single ion monitoring (SIM) mass chromatograms at the relevant $\mathrm{m} / \mathrm{z}$ to compare each analyte to its meso internal standard. Linear calibration curves $\left(r^{2}>\right.$ 0.9995) were generated in Labsolutions software (version $5.87 \mathrm{sp} 1$, Shimadzu, Japan) and the limit of quantification for bile pigments in serum and exudate approximated to be 250 $\mathrm{nM}$ for all analytes.

\section{Statistical analysis}

Statistical analysis was performed using GraphPad Prism version 8.1. Differences in leukocyte counts, cytokines and oxidative stress/damage markers were analysed via oneway ANOVA (Holm-Sidak post hoc) to test for differences between MSU only and treatment groups at each time point. Normality of data sets was assessed using the Shapiro-Wilk test. If data sets lacked normal distribution, equivalent non-parametric tests were conducted and outliers were removed using the ROUT test $(Q=1 \%)$. All error bars are displayed as SEM throughout. 


\section{Results}

An acute, sterile inflammatory response was observed in the air pouch of rats following the administration of MSU crystals, which was assessed by measuring leukocyte infiltration, cytokine concentrations and markers of oxidative stress. The population of the infiltrating leukocytes primarily consisted of neutrophils and monocytes, which peaked at 12 and $48 \mathrm{~h}$ respectively (Figures 2 and 3 ).

\section{Leukocyte infiltration}

Leukocyte infiltration was determined by measuring leukocyte counts in the pouch exudate at each timepoint via haemocytometer (Figures 2 and 3). A marked and significant $(p<0.05)$ increase in leukocyte infiltration was observed for the MSU only group when compared with the sham group from 6 to $48 \mathrm{~h}$ inclusive (Figure 2). All treatment groups (BRS, BV and ibuprofen) had significantly decreased total leukocyte and neutrophil counts compared with the MSU only group from 6 to $48 \mathrm{~h}$ inclusive (Figure 2). 

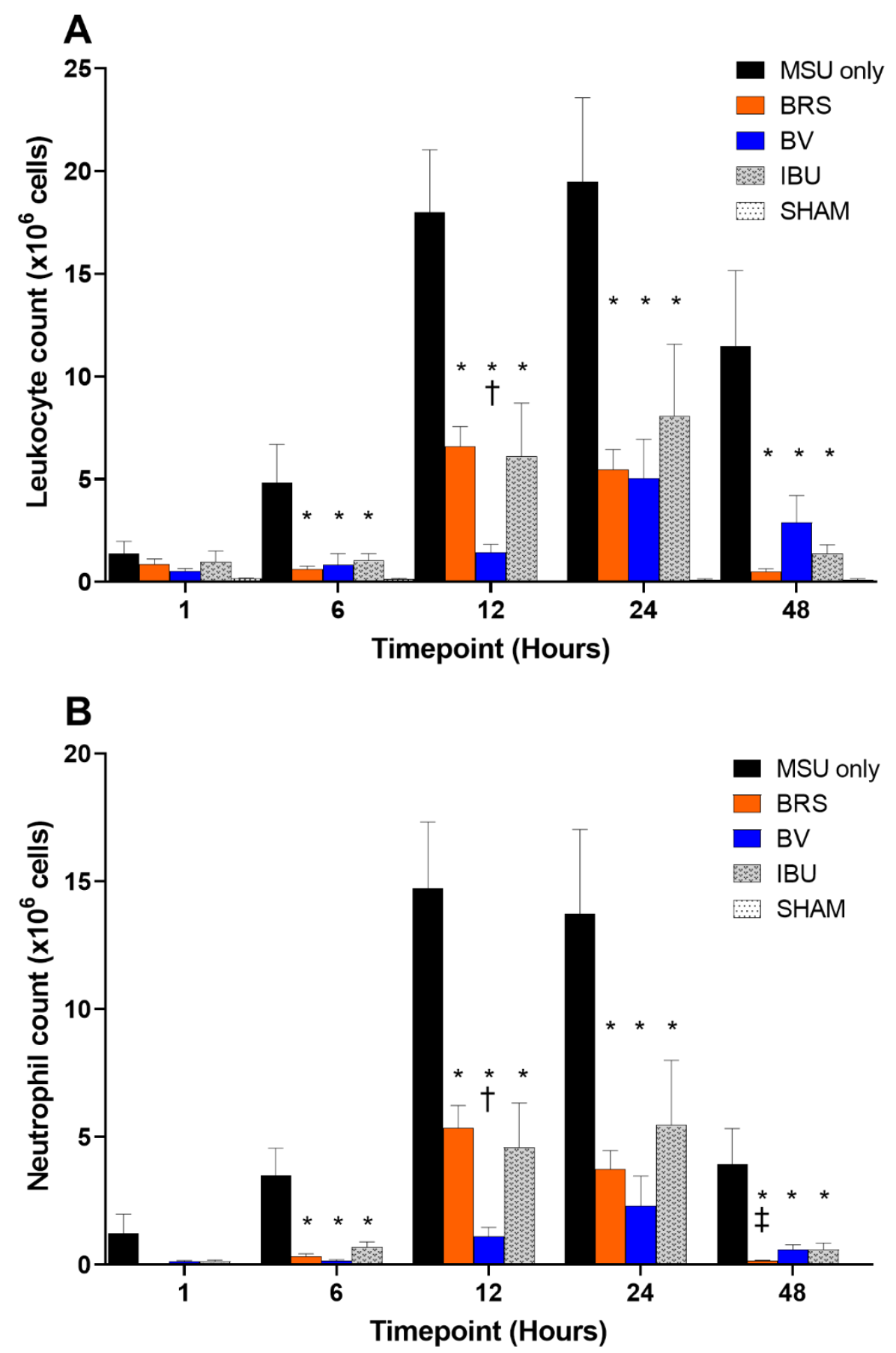

Figure 2: Effects of $27 \mathrm{mg} \mathrm{kg}^{-1}$ i.p. pre-treatment of $B R S(n=6$, orange) and $B V(n=5$, blue) on leukocyte infiltration (A) and neutrophil infiltration (B) following MSU administration ( $25 \mathrm{mg}$ in $5 \mathrm{~mL}$ sterile saline) to a 6 day old air pouch model of acute inflammation. Ibuprofen ( $8.11 \mathrm{mg} \mathrm{kg}^{-1}$ i.p. molar equivalent to BRS) was administered as a positive control (IBU $n=5$, grey), and PBS ( $3.5 \mathrm{~mL}$, i.p.) was administered as a negative control (MSU only $n=5$, black). The sham group (SHAM, white) was pre-treated with $3.5 \mathrm{~mL}$ PBS i.p. and received $5 \mathrm{~mL}$ of saline into the pouch instead of MSU. * denotes significant difference $(p<0.05)$ compared to MSU only, $\dagger$ denotes significant difference $(p<0.05)$ compared to BRS at the same timepoint and $\ddagger$ denotes significant difference $(p<0.05)$ compared to BV for the same time point. From $6-48 \mathrm{~h}$, the sham group was significantly lower than MSU only (not shown with symbol for neatness). Data are presented as mean \pm SEM. 

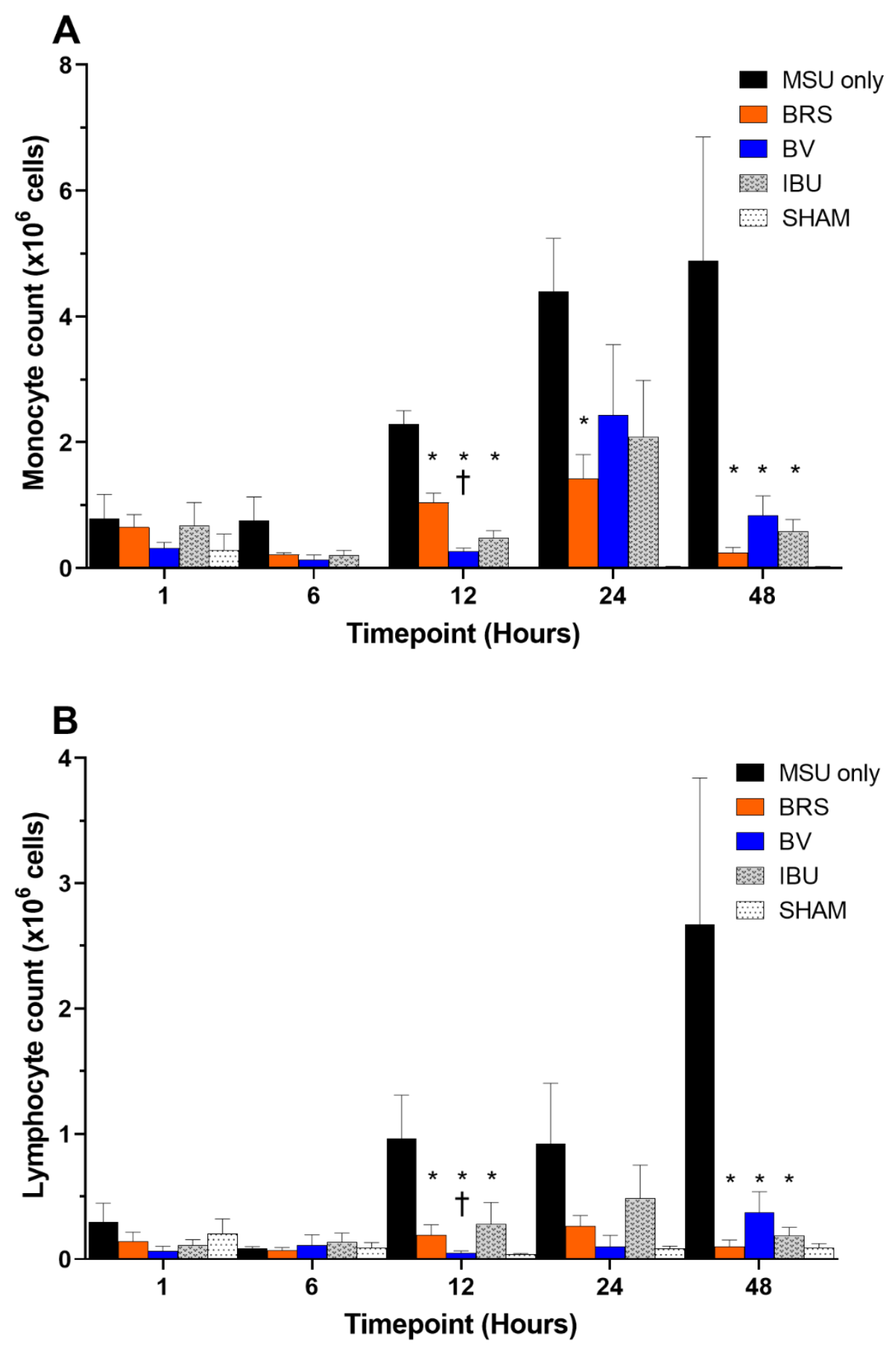

Figure 3: Effects of $27 \mathrm{mg} \mathrm{kg}^{-1}$ i.p. pre-treatment of $B R S(n=6$, orange) and $B V(n=5$, blue) on monocyte infiltration (A) and lymphocyte infiltration (B) following MSU administration ( $25 \mathrm{mg}$ in $5 \mathrm{~mL}$ sterile saline) to a 6 day old air pouch model of acute inflammation. Ibuprofen ( $8.11 \mathrm{mg} \mathrm{kg}^{-1}$ i.p., molar equivalent to BRS) was administered as a positive control (IBU $n=5$, grey), and PBS ( $3.5 \mathrm{~mL}$, i.p. $n=3$ ) was administered as a negative control (MSU only, $n=5$, black). * denotes significant difference $(p<0.05)$ compared with VEH, + denotes significant difference $(p<0.05)$ compared to BRS. From $12-48 \mathrm{~h}$, the sham group was significantly lower than MSU only (not shown with symbol for neatness). Data are presented as mean \pm SEM. 


\section{Inflammatory Cytokines}

Concentrations of cytokines in pouch exudate at $6 \mathrm{~h}$ were determined using a multiplexed bead-based flow cytometric immunoassay. Only cytokines that were significantly $(p<0.05$; unpaired t-test) elevated in MSU only groups versus sham and significantly decreased in treatment groups versus MSU only ( $p<0.05$; ANOVA) are reported here (Figure 4). All other cytokine responses can be found in Supplementary Figure 1.
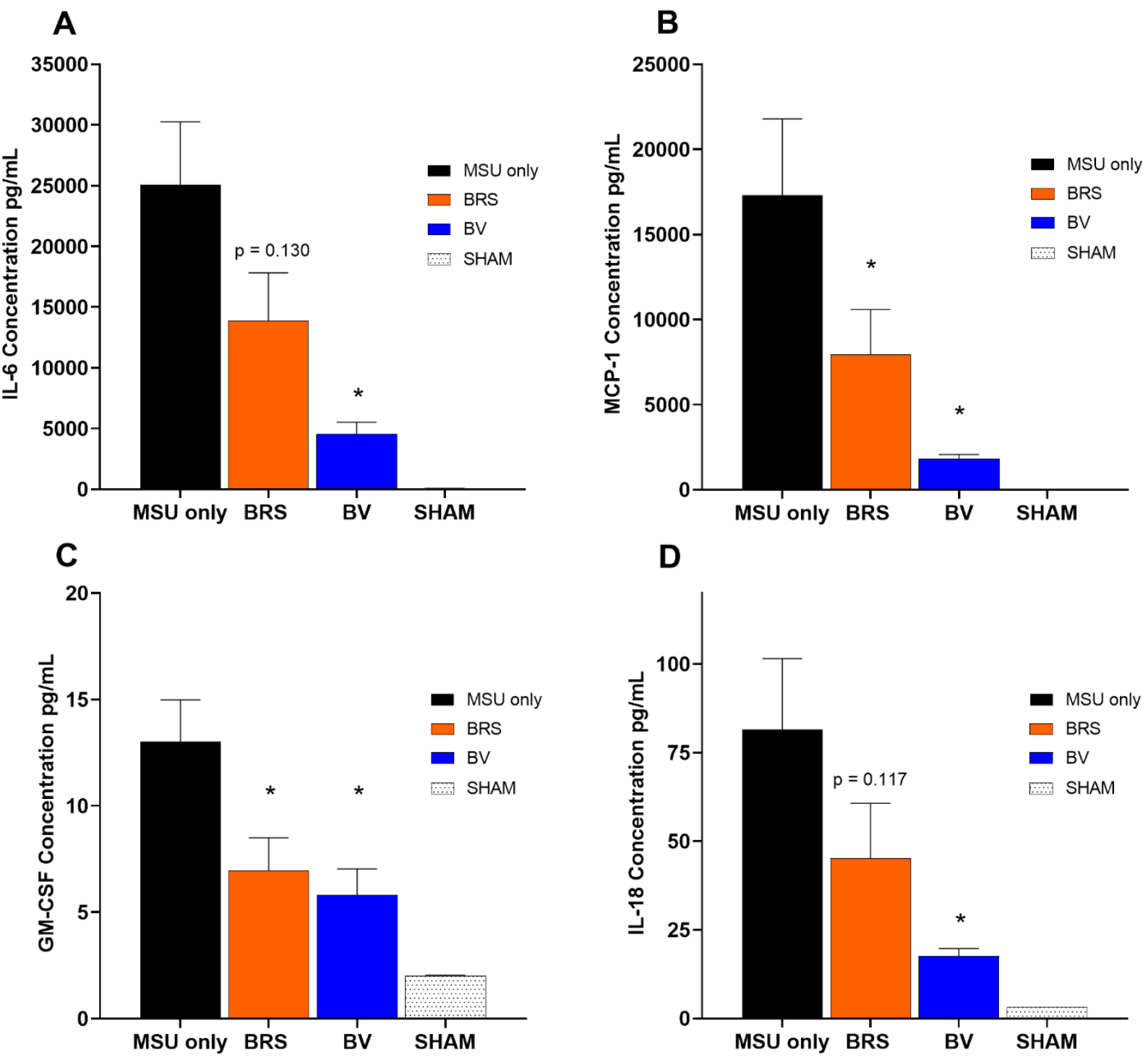

Figure 4: Effects of $27 \mathrm{mg} \mathrm{kg}^{-1}$ i.p. pre-treatment of $B R S(n=6$, orange) and $B V(n=5$, blue) on concentrations of IL-6 (A), MCP-1 (B), GM-CSF (C) and IL-18 (D) in pouch exudate following MSU administration (25 mg in $5 \mathrm{~mL}$ sterile saline) to a 6 day old air pouch model of acute inflammation at the $6 \mathrm{~h}$ time point. * denotes $p<0.05$ (ANOVA) compared with MSU only (black). Data are presented as mean \pm SEM. 


\section{Markers of Oxidative Stress}

Chloramine and protein carbonyl concentrations in BRS and BV treated animals were not significantly different when compared to the MSU only (Figure 5 and 6).

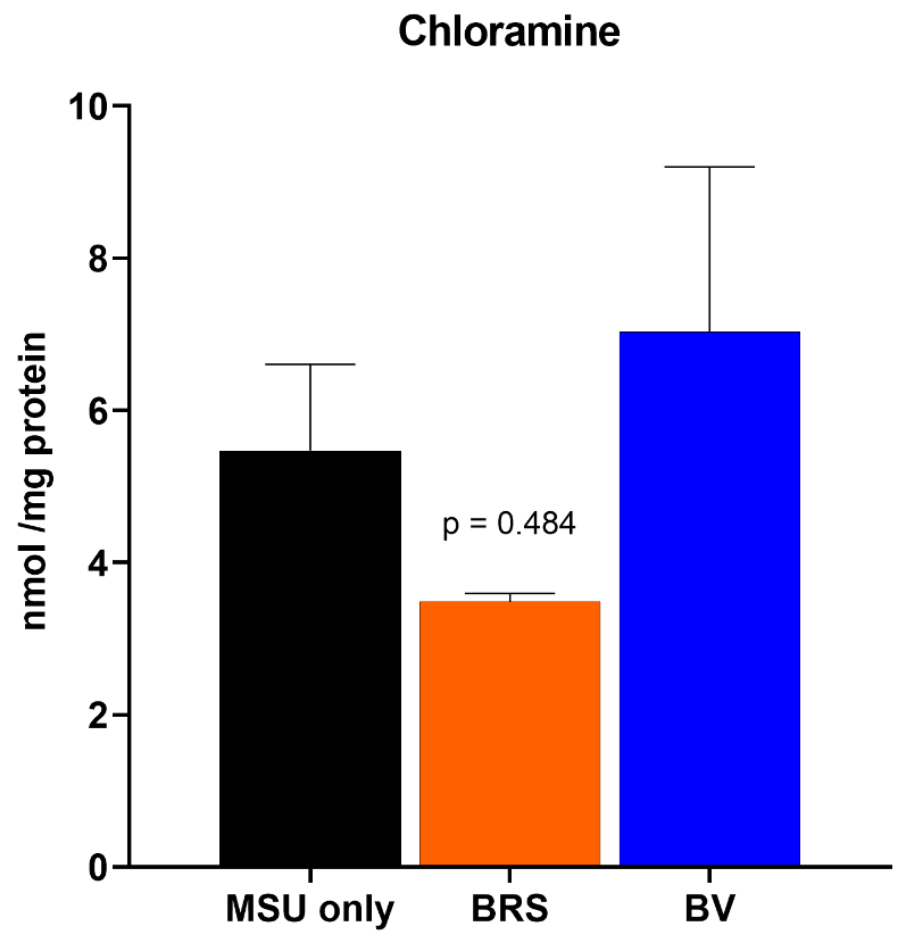

Figure 5: Effects of $27 \mathrm{mg} \mathrm{kg}^{-1}$ i.p. pre-treatment with MSU only ( $n=5$, black), BRS ( $n=6$, orange) and BV ( $n=5$, blue) on concentrations of chloramine expressed in nmol chloramine per $\mathrm{mg}$ of protein in pouch exudate following MSU administration ( $25 \mathrm{mg}$ in $5 \mathrm{~mL}$ sterile saline) into the 6 day old air pouch at the $24 \mathrm{~h}$ time point. No significant differences were detected between groups. Data are presented as mean \pm SEM. 


\section{Protein Carbonyl}

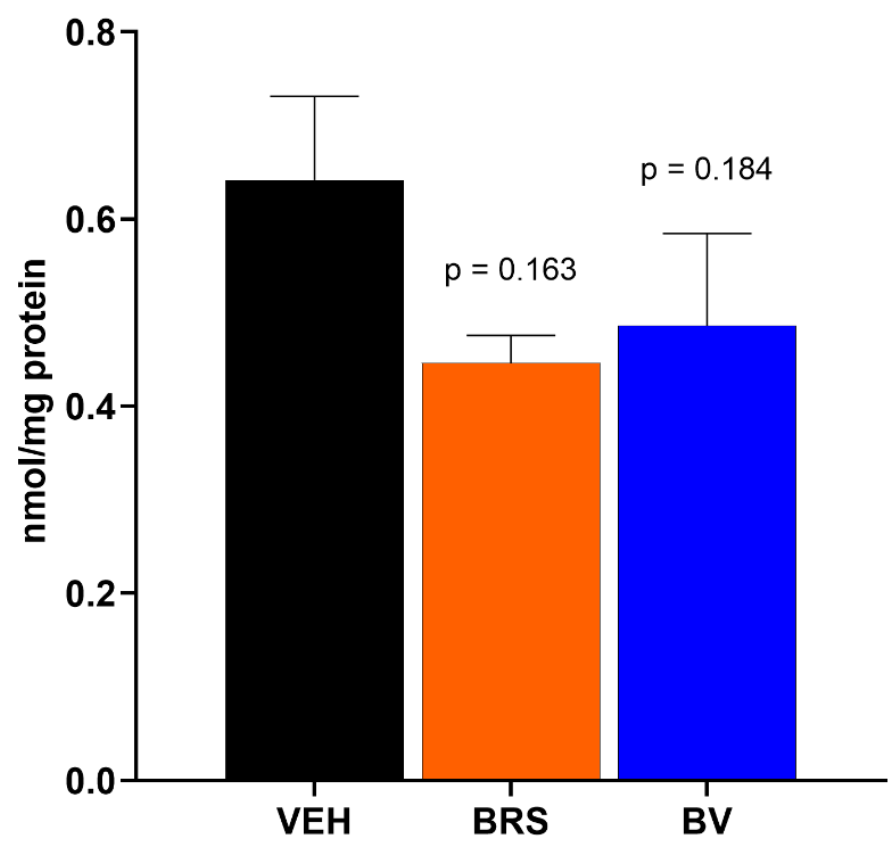

Figure 6: Effects of $27 \mathrm{mg} \mathrm{kg}^{-1}$ i.p. pre-treatment with MSU only ( $n=5$, black), BRS ( $n=6$, orange) and BV ( $n=5$, blue) on concentrations of protein carbonyl ( $\mathrm{nmol}$ carbonyl per $\mathrm{mg}$ of protein) in pouch exudate following MSU administration ( $25 \mathrm{mg}$ in $5 \mathrm{~mL}$ sterile saline) into the a 6 day old air pouch. Data are presented as mean \pm SEM.

\section{Bile Pigment Concentrations}

Concentrations of BRS, BV and UCB were determined in venous blood over the course of 48 $\mathrm{h}$ and are shown in Figure 7. 

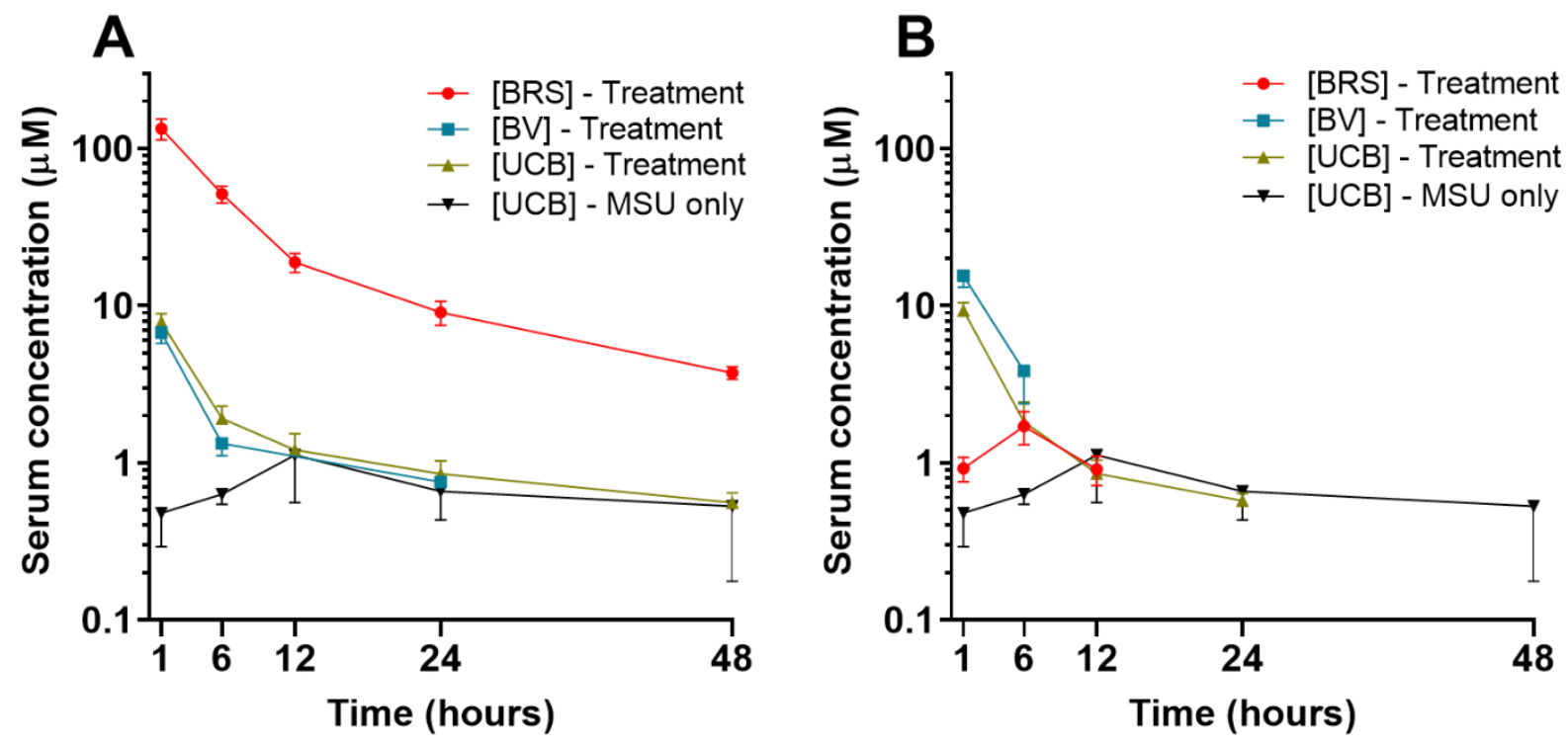

Figure 5: Effects of $27 \mathrm{mg} \mathrm{kg}^{-1}$ i.p. pre-treatment of $B R S(n=6, A$, left) and $B V(n=5, B$, right) on serum concentrations of BRS, BV and UCB. Data is omitted where compound concentrations were not detectable. Data are presented as mean \pm SEM.

Samples of pouch exudate were also analysed for bile pigment concentrations. No bile pigments were detected in the pouch exudate of MSU only or BV treated animals, however BRS was detected in pouch exudate of BRS treated animals between 1 and $24 \mathrm{~h}$ of MSU administration (Figure 8). 


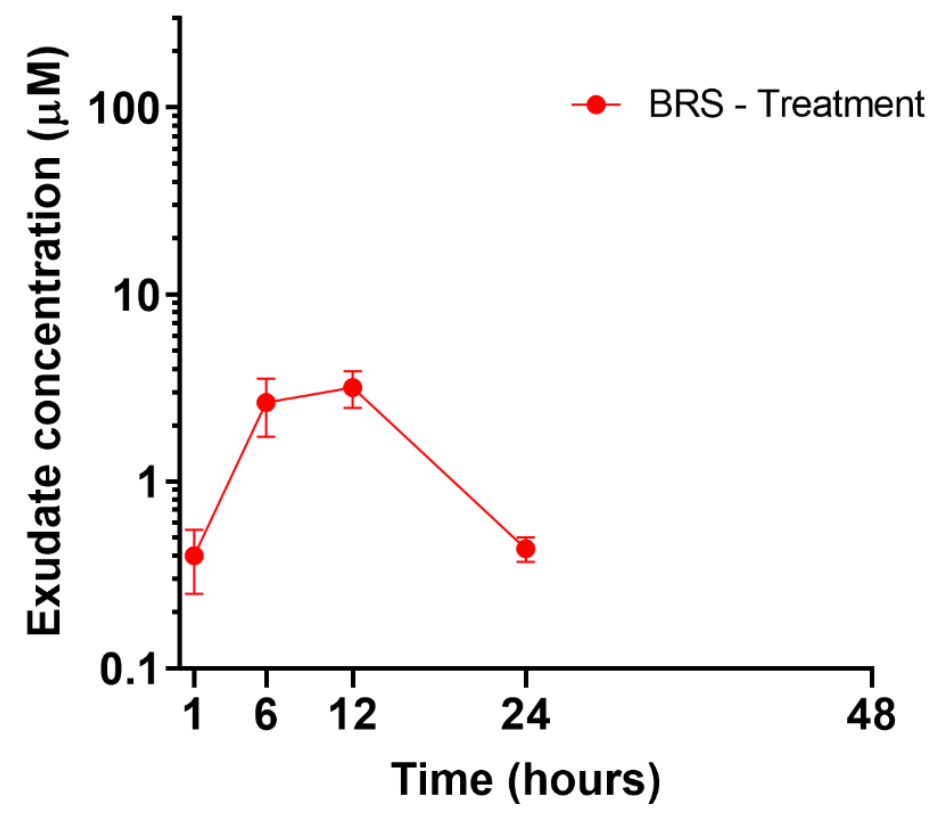

Figure 6: Effects of $27 \mathrm{mg} \mathrm{kg}^{-1}$ i.p. BRS pretreatment of $(n=6)$ on exudate BRS concentrations. BV and MSU only treatment had no quantifiable bile pigment concentrations.

\section{Discussion}

The role of inflammation in chronic disease is well established, and bile pigments continue to show therapeutic potential across a range of models of non-sterile, sterile inflammation and oxidative damage. $(4,11,20,21,32,33)$ This manuscript is the first to explore the antiinflammatory potential of bile pigment administration against gouty inflammation, and the first to describe the anti-inflammatory activity of BRS. BV and BRS pre-treatment decreased leukocyte infiltration (Figure 2A), neutrophil and monocyte ingress (Figure 2B and $3 \mathrm{~A}$ ) from 6-48 $\mathrm{h}$ and cytokine concentrations in pouch exudate (IL-6, MCP-1, GM-CSF and IL-18; Figure $4 \mathrm{~A}-\mathrm{D})$ at $6 \mathrm{~h}$ following treatment compared with the MSU only group. A large, yet select, number of cytokines were measured in this study and will assist in clarifying relevant mechanisms of action of biliverdin and bilirubin in future studies. Additionally, these data show that BRS is retained in the circulation, peaking at approximately $120 \mu \mathrm{M}$ and remaining above $5 \mu \mathrm{M}$ after $48 \mathrm{~h}$, demonstrating a favourable pharmacokinetic profile and potential to confer protection over a greater time period compared to BV.

\section{Leukocyte Infiltration}


In addition to the significant decrease in leukocyte and neutrophil infiltration observed in the BV and BRS treatment groups, monocyte and lymphocyte infiltration into the pouch from 12-48 $\mathrm{h}$ were also significantly decreased (Figure 3 ). These data suggest initial suppression of the inflammatory cascade during the early phase of the inflammatory response may have reduced the severity of subsequent leukocyte infiltration promoting prompt resolution. At $12 \mathrm{~h}$, the neutrophil (Figure 2A) and monocyte (Figure 3B) counts were significantly reduced in the BV group compared to BRS, suggesting more potent inhibition of leukocyte infiltration by BV. Interestingly, at $48 \mathrm{~h}$ BV and BRS both significantly reduced leukocyte infiltration compared with MSU only (Figures 2 and 3) and were similar with the effect observed with ibuprofen treatment, suggesting the capacity to acutely resolve inflammation is shared by both compounds and has a similar effect to that of ibuprofen. Furthermore, BRS significantly reduced neutrophil infiltration compared with BV at $48 \mathrm{~h}$ (Figure 2B), indicating that BRS administration may have been more effective in resolving and preventing neutrophil infiltration, or promoted neutrophil apoptosis/phagocytosis after $48 \mathrm{~h}$ compared to BV. Neutrophil infiltration into tissues during an inflammatory response occurs following rolling, adhesion and transmigration of neutrophils on endothelial cells. ${ }^{(34)}$ Significant down-regulation of these events occurs following BV administration in adult Balb/c mice (i.p. $9 \mathrm{mg} \mathrm{kg}^{-1}$ ). ${ }^{(34)} \mathrm{BV}$ pre-treatment in swine (single bolus i.v. $50 \mu \mathrm{mol} \mathrm{kg}{ }^{-1}$ ) also significantly inhibits neutrophil infiltration following hepatic ischaemic reperfusion injury. ${ }^{(15)}$ Furthermore, in vitro studies conclude that binding of BV to biliverdin reductase-A (BVR-A) on the surface of neutrophils and macrophages interferes with TLR4, PI3 kinase and Akt signalling pathways, thus reducing leukocyte infiltration via inhibition of chemotaxis. ${ }^{(35-39)}$ Further studies show that BVR deletion enhances macrophage chemotaxis, demonstrating an inhibitory role for BVR-A. ${ }^{(38)}$ While there are no previous data regarding BRS, UCB is reported to inhibit leukocyte extravasation with no changes observed in ICAM-1 or VCAM-1 expression. ${ }^{(40)}$ These data imply that UCB may have interfered with adhesion molecule signalling and contributed to the improved resolution seen here after $48 \mathrm{~h}$. These data further corroborate BV's inhibitory effects on chemotaxis and demonstrate, for the first time, that BRS also exhibits similar effects which in part, may occur following the spontaneous decomposition of BRS to BV. ${ }^{(20)}$

\section{Inflammatory Cytokines}


These data show that BV treatment significantly reduces IL-6, MCP-1, GM-CSF and IL-18 proinflammatory cytokine concentrations in pouch exudate $6 \mathrm{~h}$ post treatment (Figure 3) and BRS administration reduced MCP-1 and GM-CSF concentrations ( $p<0.05$; Figure 3).

Inflammatory cytokines that are clinically associated with gout-induced inflammation ${ }^{(41)}$ were decreased following BV treatment. These data supported the hypothesis and agree with published literature reporting administration of BV generally reduces pro-inflammatory cytokine expression. For example, $50 \mathrm{mg} \mathrm{kg}^{-1}$ i.p. BV treatment prior to and immediately after small bowel transplant in Lewis rats significantly decreases serum IL-6. ${ }^{(42)}$ Another study reports significant decreases in IL- 6 and MCP-1 for i.p. BV (5 mg kg ${ }^{-1}$ ) treatment in a caecal ligation model $^{(43)}$, with BV treatment delivered 8, 6, and $3 \mathrm{~h}$ before laparotomy, once immediately prior to laparotomy closure and $15 \mathrm{~h}$ postoperatively. This study repeatedly dosed BV, to ensure bioavailability of BV over a longer period, which was achieved in our study following single BRS treatment (Figure 7A). The decrease in MCP-1, a mediator of monocyte and lymphocyte recruitment associated with inflammation/insulin resistance ${ }^{(44)}$, observed in both BV and BRS administered conditions here and in the caecal ligation study ${ }^{(43)}$, support the decrease in monocyte and lymphocyte recruitment (Figure 3).

The significant decrease in IL-18 following BV treatment is a novel finding of this study. Canonically, IL-18 is an inducing factor for interferon gamma (IFN- $\gamma)^{(45)}$, suggesting that BV might have inhibit macrophage M1 polarisation as recently shown in BVR knockout macrophages. ${ }^{(39)}$ The IL-18 receptor exerts similar effects to the IL-1 receptor and plays an important role in regulating the differentiation of cell types of the adaptive immune system $^{(46)}$ Interestingly, serum IL-18 concentrations are significantly reduced in aged UGT1A1-deficient Gunn rats (i.e. rats with naturally elevated serum UCB) compared to the wild type. ${ }^{(47)}$ Biliverdin is rapidly reduced to $U C B$ in vivo (Figure $7 B$ ), therefore, it is possible that physiological reduction of BV mediated the IL-18 response in this study.

Finally, GM-CSF, a mediator of neutrophil recruitment ${ }^{(48)}$, was significantly reduced in pouch exudate at $6 \mathrm{~h}$ (Figure 4C) for BV and BRS, which supports the decreased leukocyte infiltration findings here. Additionally, a recent in vitro study reports that proinflammatory cytokine production, including GM-CSF, is downregulated by BV and phycocyanobilin (PCB; 
an algal linear tetrapyrrole structurally similar to BV) pre-treatment of human peripheral blood mononuclear cells (PBMC) stimulated by a mixed lymphocyte reaction. ${ }^{(49)}$

These data agree with recent reports that BV and enzymes involved in bile pigment metabolism (BVR/Haem Oxygenase-1) inhibit sterile inflammatory responses. For example, BV treatment protects rats from acetaminophen-induced toxicity ${ }^{(50)}$, ameliorates cerebral ischemia reperfusion injury in rats $^{(51)}$ and supresses inflammatory cytokine release in ischemia reperfusion injury and insulin resistance models. ${ }^{(52)}$ These studies, while they measure different outcomes, add to a growing body of evidence demonstrating that these bile pigments play a significant role in attenuating inflammation. Improved understanding of these compounds' roles within mammalian physiology could lead to their future development as safe and effective anti-inflammatory therapeutics.

\section{Bile Pigments in Serum}

Biliverdin administration induces potent anti-inflammatory effects ${ }^{(12-14,17)}$; however, we recently demonstrated that i.p. and i.d. biliverdin administration results in appreciable bilirubin-10-sulfonate ( 22\%) conversion in vitro and ex vivo. ${ }^{(20)}$ Following i.p. BV and BRS administration, bile pigments were systemically and readily available during the acute phase of inflammation. In addition, this manuscript brings very important information regarding longer term systemic concentrations of BRS retained in the circulation. In conscious animals, circulating concentrations ranged between 120 and $5 \mu \mathrm{M}$ over $48 \mathrm{~h}$. While BV rapidly decreased over time (i.e., 24 h) with consequent formation of unconjugated bilirubin (presumably due to metabolism via $\mathrm{BVR}^{(21)}$ ) and a small amount of BRS (Figure 7B). After BRS administration however, serum BRS concentrations were elevated for $48 \mathrm{~h}$, with only small amounts of BV present over $24 \mathrm{~h}$ (Figure 7A). These data demonstrate that BRS slowly decomposes, releasing $\mathrm{BV}$, which could mediate longer term anti-inflammatory effects via BVR signalling.

In our previous study, we tested the pharmacokinetics of the investigated compounds over $3 \mathrm{~h}$ only in Wistar rats where the animal was fasted, under a surgical plane of anaesthesia and had the bile duct cannulated which effectively interrupted entero-hepatic circulation. Both studies dosed Wistar rats of a similar weight and age with the same amounts of both 
compounds via the intraperitoneal route and the $1 \mathrm{~h}$ timepoints in both studies were comparable, with the peak serum concentration in both studies reaching in excess of 100 $\mu \mathrm{M}$ for $\mathrm{BRS}$ and $\sim 17 \mu \mathrm{M}$ for BV. While both experiments demonstrated a rapid decline in circulating BV after administration, BRS maintained a serum concentration of $\sim 100 \mu \mathrm{M}$ after $3 \mathrm{~h}$ (in anesthetised rats), whereas in the present study concentrations fell to $50 \mu \mathrm{M}$ after 6 $\mathrm{h}$ representing the gradual elimination of the compound from blood. As this present study administered BRS and BV to free-living and conscious animals, the pk data presented here are not influenced by the effects of anaesthetics, or interruption of the enterohepatic circulation, providing clearer data of each compound's longer-term bioavailability.

We also detected BRS in pouch exudate (Figure 8) over $24 \mathrm{~h}$, indicating that it either leaked into the pouch with exudate formation, or diffused through tissues into this compartment. Surprisingly, markers of oxidative stress were not significantly impacted by BV or BRS (despite consistent reductions in protein carbonyl for BRS and BV) (Figures 5 and 6). These data are, however, most likely related to the relatively low local concentrations of bile pigments in the pouch environment. Future studies could investigate the effect of injecting the compounds directly into the pouch (akin to the joint) to determine whether local concentrations can similarly inhibit inflammatory and oxidative processes. Additionally, MPO concentrations in pouch exudate were below the limit of detection for our assay; however, it is possible that BRS, which possesses a reduced $\mathrm{C} 10$ bridge, behaves similar to UCB, which inhibits neutrophil burst and neutralises superoxide radicals. ${ }^{(19)}$ Furthermore, high urate concentrations in the pouch may also have competed for radical formation over the relatively low concentrations of BRS, impacting protection from pouch fluid oxidation. Therefore perhaps increased bile pigment concentrations in pouch fluid could inhibit MPO mediated chloramine and protein carbonyl formation ${ }^{(53)}$, supporting the need for further studies to address this question.

\section{Conclusion}

Current human and clinical data indicates consistent benefits associated with mild elevation of UCB (as observed in Gilbert's Syndrome) as well as the potential of tetrapyrrole administration ${ }^{(32)}$ to treat a myriad of inflammatory diseases including cardiovascular disease and type 2 diabetes mellitus. 
This study describes the in vivo anti-inflammatory efficacy of BRS and BV in an MSU crystal induced model of sterile inflammation in the rat, as well as longer term serum pharmacokinetics in conscious animals for both compounds. The data presented demonstrates the importance of bile pigments as endogenous anti-inflammatory compounds that are actively produced during sterile inflammation and indicates that BV/BRS administration are potential therapeutic avenues for the treatment of sterile inflammation/gout. BRS administration confers similar benefits to BV administration while remaining in systemic circulation for up to $48 \mathrm{~h}$ following treatment. We propose that the administration of BRS, a hydrophilic compound, is a simple method to increase BV and UCB in vivo, which could help in realising the anti-inflammatory therapeutic potential of bile pigments.

\section{Credit Statement}

Ryan G. Shiels: Conceptualization, Methodology, Validation, Formal analysis, Writing - Original Draft, Writing - Review \& Editing, Visualization, Project administration. Wenu Hewage: Investigation, Writing - Original Draft, Writing Review \& Editing. Evan Pennell: Investigation, Writing - Review \& Editing Josif Vidimce: Investigation, Writing - Review \& Editing. Andrew G. Pearson: Writing Review \& Editing, Supervision. Karl-Heinz Wagner: Writing - Review \& Editing, Supervision, Funding acquisition. Michael Morgan: Methodology, Writing - Review \& Editing, Supervision Andrew C. Bulmer: Conceptualization, Methodology, Investigation, Writing - Review \& Editing, Supervision, Project administration, Funding acquisition.

\section{Author contribution statement}

A.C.B., M.M. and R.G.S. conceived the design of this study. R.G.S. and W.H. performed animal experimentation and interpretation of data. R.G.S. and W.H. performed HPLC and LCMS analysis, pouch exudate analysis and statistical analysis of data. J.V. assisted with statistical analysis and performed quantification of protein carbonyl. E.N.P. assisted with the quantification of cytokines via flow cytometry. A.G.P., G.G. and K.H.W. assisted with concept, technical advice and input. A.C.B. and K.H.W. secured funding for the project. All authors contributed to revision of the MS and provided their final approval for manuscript submission.

\section{Competing interests}

The author(s) declare no competing interests.

\section{Acknowledgement}


The authors acknowledge the support received through an Australian Government Research Training Program Scholarship as well as the generosity of Patricia Barlow in providing a Research Higher Degree scholarship. The research was supported by the Griffith Enterprise Innovation Fund, which utilised funding provided by Griffith University and the Queensland Government.

\section{Animal Use}

All animal care and experimental procedures complied with the Guidelines of the Australian National Health and Medical Research Council and were approved by the Griffith University Animal Ethics Committee (MSC/03/18/AEC).

Supplementary information

Supplementary Figure 7: Concentrations of cytokines in pouch exudate at $6 \mathrm{~h}$ were determined using a multiplexed bead-based flow cytometric immunoassay.

\section{References}

1. Busch, A.W. and B.L. Montgomery (2015). Interdependence of tetrapyrrole metabolism, the generation of oxidative stress and the mitigative oxidative stress response. Redox Biol. 4: p. 260-71.

2. McDonagh, A.F. Bile pigments: Bilatrienes and 5,15-biladienes. The Porphyrins, ed. D. D. Vol. VI. 1979, New York: Academic.

3. Vitek, L., et al. (2006). Identification of bilirubin reduction products formed by Clostridium perfringens isolated from human neonatal fecal flora. J Chromatogr $B$ Analyt Technol Biomed Life Sci. 833(2): p. 149-57.

4. Bulmer, A.C., Blanchfield, J.T., Riedc, K. \& Wagner, K.H. (2008). The anti-mutagenic properties of bile pigment. Reviews in Mutation Research. 658(1): p. 28-41.

5. Bulmer, A.C., et al. (2018). Bilirubin acts as a multipotent guardian of cardiovascular integrity: more than just a radical idea. (1522-1539 (Electronic)).

6. Bellner, L., et al. (2011). Biliverdin Rescues the HO-2 Null Mouse Phenotype of Unresolved Chronic Inflammation Following Corneal Epithelial Injury. Invest Ophthalmol Vis Sci. 52(6): p. 3246-53.

7. Vitek, L.N., L. (2003). Inverse relationship between serum bilirubin and atherosclerosis in men: a meta-analysis of published studies. Experimental Biology \& Medicine. 228(5): p. 568-571.

8. Stocker, R. and J.F. Keaney, Jr. (2004). Role of oxidative modifications in atherosclerosis. Physiol Rev. 84(4): p. 1381-478.

9. Bulmer, A.A.-O., et al. (2018). Bilirubin acts as a multipotent guardian of cardiovascular integrity: more than just a radical idea. (1522-1539 (Electronic)).

10. Wagner, K.H., et al. (2018). Diagnostic criteria and contributors to Gilbert's syndrome. Crit Rev Clin Lab Sci. 55(2): p. 129-139. 
11. Bulmer, A.C., Blanchfield, J.T., Toth, I.F., \& Coombes, J.S. (2008). Improved resistance to serum oxidation in Gilbert's Syndrome; a mechanism for cardioprotection.

Atherosclerosis. 199(2): p. 390-396.

12. Nakagami, T., Toyomura, K., Kinoshita, T. \& Morisawa, S. (1993). A beneficial role of bile pigments as an endogenous tissue protector: Anti-complement effects of biliverdin and conjugated bilirubin. Biochimica et Biophysica Acta-General Subjects. 1158(2): p. 189-193.

13. Nakao, A., et al. (2005). Biliverdin administration prevents the formation of intimal hyperplasia induced by vascular injury. Circulation. 112(4): p. 587-91.

14. Sarady-Andrews, J.K., et al. (2005). Biliverdin administration protects against endotoxin-induced acute lung injury in rats. Am J Physiol Lung Cell Mol Physiol. 289(6): p. L1131-7.

15. Andria, B., et al. (2013). Biliverdin protects against liver ischemia reperfusion injury in swine. PLoS One. 8(7): p. e69972.

16. Bakrania, B., et al. (2016). Pre- or post-ischemic bilirubin ditaurate treatment reduces oxidative tissue damage and improves cardiac function. Int J Cardiol. 202: p. 27-33.

17. Fondevila, C., et al. (2004). Biliverdin therapy protects rat livers from ischemia and reperfusion injury. Hepatology. 40(6): p. 1333-41.

18. Nakagami, T., et al. (1993). A beneficial role of bile pigments as an endogenous tissue protector: Anti-complement effects of biliverdin and conjugated bilirubin. Biochimica et Biophysica Acta (BBA) - General Subjects. 1158(2): p. 189-193.

19. Vasavda, C., et al. (2019). Bilirubin Links Heme Metabolism to Neuroprotection by Scavenging Superoxide. Cell Chem Biol. 26(10): p. 1450-1460 e7.

20. Shiels, R.G., et al. (2019). Unprecedented Microbial Conversion of Biliverdin into Bilirubin-10-sulfonate. Sci Rep. 9(1): p. 2988.

21. Bulmer, A.C., et al. (2011). Bile pigment pharmacokinetics and absorption in the rat: therapeutic potential for enteral administration. Br J Pharmacol. 164(7): p. 1857-70.

22. Li, C., et al. (2013). Ambulatory Resource Utilization and Cost for Gout in United States. The American Journal of Pharmacy Benefits. 5(2): p. e46 - e54.

23. Orlowski, G.M., et al. (2015). Multiple Cathepsins Promote Pro-IL-1beta Synthesis and NLRP3-Mediated IL-1beta Activation. J Immunol. 195(4): p. 1685-97.

24. Jaramillo, M., P.H. Naccache, and M. Olivier (2004). Monosodium urate crystals synergize with IFN-gamma to generate macrophage nitric oxide: involvement of extracellular signal-regulated kinase $1 / 2$ and NF-kappa B. J Immunol. 172(9): p. 573442.

25. Brooks, P.M., D. Burton, and M.J. Forrest (1987). Crystal-induced inflammation in the rat subcutaneous air-pouch. Br J Pharmacol. 90(2): p. 413-9.

26. Chapman, P.T., et al. (1997). Endothelial activation in monosodium urate monohydrate crystal-induced inflammation: in vitro and in vivo studies on the roles of tumor necrosis factor alpha and interleukin-1. Arthritis Rheum. 40(5): p. 955-65.

27. Busso, N. and A. So (2010). Mechanisms of inflammation in gout. Arthritis Res Ther. 12(2): p. 206.

28. McCarty, D.J., Jr. (1965). The inflammatory reaction to microcrystalline sodium urate. Arthritis Rheum. 8(5): p. 726-35.

29. Fam, A.G., et al. (1992). A comparison of five preparations of synthetic monosodium urate monohydrate crystals. J Rheumatol. 19(5): p. 780-7. 
30. Martin, S.W., et al. (1994). The six-day-old rat air pouch model of inflammation: characterization of the inflammatory response to carrageenan. J Pharmacol Toxicol Methods. 32(3): p. 139-47.

31. Dypbukt, J.M., et al. (2005). A sensitive and selective assay for chloramine production by myeloperoxidase. Free Radic Biol Med. 39(11): p. 1468-77.

32. Vitek, L., C. Bellarosa, and C. Tiribelli (2019). Induction of Mild Hyperbilirubinemia: Hype or Real Therapeutic Opportunity? Clin Pharmacol Ther. 106(3): p. 568-575.

33. Bulmer, A.C., H.J. Verkade, and K.H. Wagner (2013). Bilirubin and beyond: a review of lipid status in Gilbert's syndrome and its relevance to cardiovascular disease protection. Prog Lipid Res. 52(2): p. 193-205.

34. Freitas, A., et al. (2006). Heme oxygenase/carbon monoxide-biliverdin pathway down regulates neutrophil rolling, adhesion and migration in acute inflammation. $\mathrm{Br}$ J Pharmacol. 149(4): p. 345-54.

35. Ishikawa, K., et al. (1997). Induction of heme oxygenase-1 inhibits the monocyte transmigration induced by mildly oxidized LDL. J Clin Invest. 100(5): p. 1209-16.

36. Wegiel, B. and L.E. Otterbein (2012). Go green: the anti-inflammatory effects of biliverdin reductase. Front Pharmacol. 3(1): p. 47.

37. Wegiel, B., et al. (2011). Biliverdin inhibits Toll-like receptor-4 (TLR4) expression through nitric oxide-dependent nuclear translocation of biliverdin reductase. Proc Natl Acad Sci U S A. 108(46): p. 18849-54.

38. Bisht, K., et al. (2019). Deletion of Biliverdin Reductase A in Myeloid Cells Promotes Chemokine Expression and Chemotaxis in Part via a Complement C5a--C5aR1 Pathway. J Immunol. 202(10): p. 2982-2990.

39. Bisht, K., et al. (2014). Biliverdin modulates the expression of C5aR in response to endotoxin in part via mTOR signaling. Biochem Biophys Res Commun. 449(1): p. 94-9.

40. Vogel, M.E., et al. (2017). Bilirubin Prevents Atherosclerotic Lesion Formation in LowDensity Lipoprotein Receptor-Deficient Mice by Inhibiting Endothelial VCAM-1 and ICAM-1 Signaling. J Am Heart Assoc. 6(4): p. e004820.

41. Cavalcanti, N.G., et al. (2016). Cytokine Profile in Gout: Inflammation Driven by IL-6 and IL-18? Immunol Invest. 45(5): p. 383-95.

42. Nakao, A., et al. (2004). Biliverdin protects the functional integrity of a transplanted syngeneic small bowel. Gastroenterology. 127(2): p. 595-606.

43. Overhaus, M., et al. (2006). Biliverdin protects against polymicrobial sepsis by modulating inflammatory mediators. Am J Physiol Gastrointest Liver Physiol. 290(4): p. G695-703.

44. Cranford, T.L., et al. (2016). Role of MCP-1 on inflammatory processes and metabolic dysfunction following high-fat feedings in the FVB/N strain. Int J Obes (Lond). 40(5): p. 844-51.

45. Yasuda, K., K. Nakanishi, and H. Tsutsui (2019). Interleukin-18 in Health and Disease. Int J Mol Sci. 20(3): p. 649.

46. Zaki, M.H., et al. (2010). IL-18 production downstream of the NIrp3 inflammasome confers protection against colorectal tumor formation. J Immunol. 185(8): p. 491220.

47. Zelenka, J., et al. (2016). Hyperbilirubinemia Protects against Aging-Associated Inflammation and Metabolic Deterioration. Oxid Med Cell Longev. 2016: p. 6190609.

48. Mehta, H.M., M. Malandra, and S.J. Corey (2015). G-CSF and GM-CSF in Neutropenia. J Immunol. 195(4): p. 1341-9. 
49. Basdeo, S.A., et al. (2016). Suppression of human alloreactive T cells by linear tetrapyrroles; relevance for transplantation. Transl Res. 178(1878-1810 (Electronic)): p. 81-94 e2.

50. Chiu, H., J.A. Brittingham, and D.L. Laskin (2002). Differential induction of heme oxygenase-1 in macrophages and hepatocytes during acetaminophen-induced hepatotoxicity in the rat: effects of hemin and biliverdin. Toxicol Appl Pharmacol. 181(2): p. 106-15.

51. Li, J.J., et al. (2017). Biliverdin administration ameliorates cerebral ischemia reperfusion injury in rats and is associated with proinflammatory factor downregulation. Exp Ther Med. 14(1): p. 671-679.

52. Takei, R., et al. (2019). Bilirubin reduces visceral obesity and insulin resistance by suppression of inflammatory cytokines. PLoS One. 14(10): p. e0223302.

53. Boon, A.C., et al. (2015). Bilirubin scavenges chloramines and inhibits myeloperoxidase-induced protein/lipid oxidation in physiologically relevant hyperbilirubinemic serum. Free Radic Biol Med. 86(1873-4596 (Electronic)): p. 25968. 